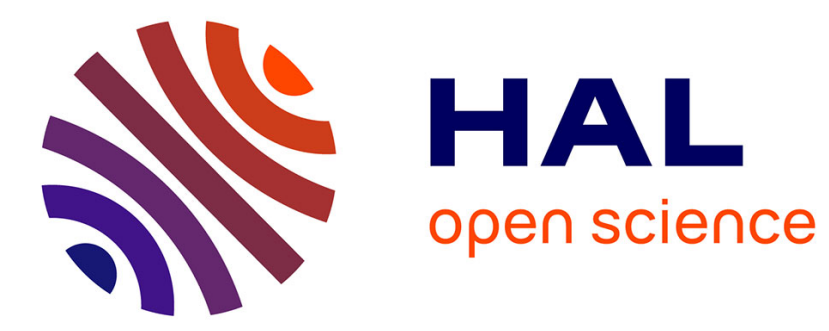

\title{
Growth Rate and Ergodicity Conditions for a Class of Random Trees
}

Guy Fayolle, Maxim Krikun

\section{To cite this version:}

Guy Fayolle, Maxim Krikun. Growth Rate and Ergodicity Conditions for a Class of Random Trees.

[Research Report] RR-4331, INRIA. 2001. inria-00072256

\section{HAL Id: inria-00072256 \\ https://hal.inria.fr/inria-00072256}

Submitted on 23 May 2006

HAL is a multi-disciplinary open access archive for the deposit and dissemination of scientific research documents, whether they are published or not. The documents may come from teaching and research institutions in France or abroad, or from public or private research centers.
L'archive ouverte pluridisciplinaire HAL, est destinée au dépôt et à la diffusion de documents scientifiques de niveau recherche, publiés ou non, émanant des établissements d'enseignement et de recherche français ou étrangers, des laboratoires publics ou privés. 


\section{Growth Rate and Ergodicity Conditions for a Class of Random Trees}

Guy Fayolle — Maxim Krikun

\section{$\mathbf{N}^{\circ} 4331$}

December 2001 THÈME 1 



\title{
Growth Rate and Ergodicity Conditions for a Class of Random Trees
}

\author{
Guy Fayolle* , Maxim Krikun ${ }^{\dagger}$ \\ Thème 1 - Réseaux et systèmes \\ Projet Meval \\ Rapport de recherche $\mathrm{n}^{\circ} 4331$ - December 2001 - 17 pages
}

\begin{abstract}
This paper gives the growth rate and the ergodicity conditions for a simple class of random trees. New edges appear according to a Poisson process, and leaves can be deleted at a rate $\mu$. The main results lay the stress on the famous number $e$. In the case of a pure birth process, i.e. $\mu=0$, the height of the tree at time $t$ grows linearly at the rate $e$, in mean and almost surely as $t \rightarrow \infty$. When deletions of leaves are permitted, a complete classification of the process is given in terms of the intensity factor $\rho=\lambda / \mu$ : it is ergodic if $\rho \leq e^{-1}$, and transient if $\rho>e^{-1}$. There is a phase transition phenomenon: the usual region of null recurrence (in the parameter space) here does not exist. This fact is rare for countable Markov chains with exponentially distributed jumps. Bounds are obtained for the transient regime.
\end{abstract}

Key-words: random trees, ergodicity, transience, non-linear differential equations.

\footnotetext{
* Guy.Fayolle@inria.fr - INRIA - Domaine de Voluceau, Rocquencourt - BP 105 - 78153 Le Chesnay Cedex - France

$\dagger$ Laboratory of Large Random Systems, Faculty of Mathematics and Mechanics, Moscow State University, 119899, Moscow - Russia
} 


\section{Taux de croissance et conditions d'ergodicité pour certains arbres aléatoires}

Résumé : Cet article donne le taux de croissante et les conditions d'ergodicité pour une classe d'abres aléatoires avec adjonction d'arcs suivant un Processus de Poisson et suppression de feuilles suivant un taux $\mu$. Les résultats principaux mettent en vedette le nombre $e$. Dans le cas de naissance pure, i.e. $\mu=0$, la hauteur d'arbre à l'instant $t$ croît linéairement avec une pente asymptotiquement égale à $e$, en moyenne et presque sûrement lorsque $t \rightarrow \infty$. Lorsque les suppressions de feuilles sont également autorisées, on obtient une classification complète du processus selon les valeurs du facteur d'intensité $\rho=\lambda / \mu$ : ergodicité si $\rho \leq e^{-1}$ et transience si $\rho>e^{-1}$. Un phénomène de transition de phase apparaît : dans l'espace des paramètres, la région correspondant habituellement à la récurrence nulle n'existe pas. Cette situation est rare pour des chaînes de Markov dénombrables dont les lois des sauts sont exponentielles. Des bornes sont également proposées dans le cas de transience.

Mots-clés : arbres aléatoires, ergodicité, transience, équations différentielles non linéaires. 


\section{Introduction}

So far, few results seem to exist for random trees as soon as insertions and deletions are simultaneously permitted (see e.g. [6]). Let $G=\{G(t), t \geq 0\}$ be a continuous time Markov chain with state space the set of finite directed trees rooted at some fixed vertex $V_{0}$. This paper is a self-contained continuation of [5] and it gives exact results about the growth rate and ergodicity conditions of $G$ under some evolution rules, which correspond to quite natural models, since one may think of a vertex as being the node of a network or of some general data structure.

\section{$1.1 \quad$ Notation}

Throughout the study, the distance between two vertices is the number of edges in the path joining them, and the height $h(V)$ of a vertex $V$ is the distance from the root. The set of vertices having the same height $k$ form the $k$-th level of the tree, the root $V_{0}$ being at level 0 . Hence the height of $G$ is a stochastic process $H_{G} \stackrel{\text { def }}{=}\left\{H_{G}(t), t \geq 0\right\}$, where

$$
H_{G}(t) \stackrel{\text { def }}{=} \max _{V \in G(t)} h(V) .
$$

Wherever the meaning is clear from the context, we shall omit the subscript $G$ and simply write $H$. The indegree of a vertex $V$ is the number of edges starting at $V$ and a vertex with indegree 0 is a leaf. Finally, we will also need the classical notion of subtree with root $V$, which goes without saying.

\subsection{The basic model}

At time $t=0, G(0)$ consists of the single vertex $V_{0}$. Then at time $t>0$, the transitions on $G$ are of two types:

- Adjunction. At each vertex $V$, a new edge having its origin at $V$ can be appended to the tree at the epoches of a Poisson process with parameter $\lambda>0$. In this case, the indegree of $V$ is increased by one and the new edge produces a new leaf. 
- Deletion. From its birth, any leaf (but the root) can be deleted at a rate $\mu$. In other words, a vertex as long as it has no descendant has an exponentially distributed lifetime with parameter $\mu \geq 0$.

In section 2 , on computes the growth rate of $H_{G}$ in the case $\mu=0$. Section 3 is devoted to the ergodicity conditions of $G$ in the general situation $\mu>0$.

\section{Pure growth: $\mu=0$}

In this section we shall only consider the pure birth process, that is $\mu=0$. Without loss of generality, we will put $\lambda=1$, since this parameter appears only as a factor of the time $t$. The main outcome is quoted now.

Theorem 2.1.

$$
\left\{\begin{array}{l}
\lim _{t \rightarrow \infty} \frac{H(t)}{t}=e, \text { with probability one } \\
\lim _{t \rightarrow \infty} \frac{\mathrm{E} H(t)}{t}=e .
\end{array}\right.
$$

The proof is a direct consequence of the four forthcoming lemmas.

Denote by $X_{n}(t)$ the number of vertices at the $n$-th level.

\section{Lemma 2.1.}

$$
\mathrm{E} X_{n}(t)=\frac{t^{n}}{n !}
$$

Proof. Since

$$
\mathrm{P}\left\{X_{n}(t+d t)=X_{n}(t)+1 \mid X_{n}(t), X_{n-1}(t)\right\}=X_{n-1}(t) d t+o(d t),
$$

we obtain

$$
\left\{\begin{array}{l}
\frac{d}{d t} \mathrm{E} X_{n}(t)=\mathrm{E} X_{n-1}(t), \quad n \geq 1, \\
\mathrm{E} X_{0}(t)=1
\end{array}\right.
$$

and the result is immediate by induction. 


\section{Lemma 2.2.}

$$
\mathrm{E} H(t) \leq e t+\frac{\sqrt{e t}}{\sqrt{2 \pi}(e-1)}
$$

Proof. Clearly, $\mathrm{P}\{H(t) \geq k\}=\mathrm{P}\left\{X_{k}(t)>0\right\} \leq \mathrm{E} X_{k}(t)$, and therefore

$$
\mathrm{E} H(t)=\sum_{k=1}^{\infty} \mathrm{P}\{H(t) \geq k\} \leq \sum_{k=1}^{m} 1+\sum_{k=m+1}^{\infty} \frac{t^{k}}{k !} .
$$

Taking $m=\lceil e t\rceil$ and using Stirling's formula we have

$$
\begin{aligned}
\mathrm{E} H(t) & \leq m+\frac{t^{m+1}}{(m+1) !} \sum_{k=0}^{\infty}\left(\frac{t}{m+1}\right)^{k} \leq e t+\left(\frac{e}{e-1}\right) \frac{t^{e t+1}}{(e t)^{e t} e^{-e t} \sqrt{2 \pi e t}} \\
& \leq e t+\frac{\sqrt{e}}{\sqrt{2 \pi}(e-1)} \sqrt{t} .
\end{aligned}
$$

Lemma 2.3. With probability one,

$$
\liminf _{t \rightarrow \infty} \frac{H(t)}{t} \geq e
$$

Proof. We introduce a so-called restricted tree, which means that any vertex of $G$ can possibly be frozen, according to some given policy. The main point is that a frozen vertex does not produce new edges. Then the corresponding restricted tree $G^{\prime}$ is a random process defined on the same probability space as $G$. From the obvious inequality $H_{G^{\prime}} \leq H_{G}$, the height of a restricted tree appears to be a lower estimate for $H_{G}$.

Fix an integer $n \geq 1$ and some time $\tau>0$. The policy used to freeze vertices will be the following: in each time interval $[\tau k, \tau(k+1)], k=0,1,2, \ldots$, only levels $n k, n k+1, \ldots, n k+(n-1)$ are allowed to produce new edges.

Let $Y_{k}^{(n)}$ be the number of vertices at the $(n k)$-th level of the restricted tree obtained at time $\tau k$. Clearly $Y_{k}^{(n)}$ is a branching process with offspring distribution $X_{n}(\tau)$. Taking $\tau$ and $n$ subject to the constraint

$$
\mathrm{E} X_{n}(\tau)=\tau^{n} / n !>1
$$

$\mathrm{RR} \mathrm{n}^{\circ} 4331$ 
i.e. $\frac{n}{\tau}<e$ for $n$ sufficiently large, we get

$$
y(\tau, n) \stackrel{\text { def }}{=} \mathrm{P}\left\{Y_{k}^{(n)}>0 \text { for all } k\right\}>0,
$$

which implies

$$
\mathrm{P}\left\{\liminf _{t \rightarrow \infty} \frac{H(t)}{t} \geq \frac{n}{\tau}\right\} \geq \mathrm{P}\{H(k \tau) \geq k n \text { for all } k\} \geq y(\tau, n)>0 .
$$

Let $N(t)$ denote the number of vertices of $G(t)$. Then given, $\varepsilon>0$, one can find $N_{0}$ and $T$, respectively, such that

$$
[1-y(\tau, n)]^{N_{0}}>1-\varepsilon, \quad P\left\{N(T)>N_{0}\right\}>1-\varepsilon,
$$

which implies that, at time $T$, there will be at least $N_{0}$ vertices with probability $(1-\varepsilon)$, and, among the restricted trees rooted at each vertex, one of them will survive with the same probability. Accordingly,

$$
\mathrm{P}\{H(T+k \tau) \geq k n \text { for all } k\}>(1-\varepsilon)^{2}
$$

and

$$
\mathrm{P}\left\{\liminf _{t \rightarrow \infty} \frac{H(t+T)}{t} \geq \frac{n}{\tau}\right\}=\mathrm{P}\left\{\liminf _{t \rightarrow \infty} \frac{H(t)}{t} \geq \frac{n}{\tau}\right\}>(1-\varepsilon)^{2} .
$$

Keeping in mind that the last inequality holds for all $\varepsilon>0$ and all $n, \tau$ with $n / \tau<e$, we can write

$$
\mathrm{P}\left\{\liminf _{t \rightarrow \infty} \frac{H(t)}{t} \geq e\right\}=1 .
$$

The proof of the lemma is terminated.

Lemma 2.4. With probability one,

$$
\limsup _{t \rightarrow \infty} \frac{H(t)}{t} \leq e
$$

Proof. Choose now $\tau$ and $n$ such that $n / \tau=\gamma e$ with $\gamma>1$. Then,

$$
\begin{aligned}
\mathrm{P}\left\{\limsup _{t \rightarrow \infty} \frac{H(t)}{t}<\gamma e\right\} & \geq \mathrm{P}\{H(k \tau)<k n, k=1,2, \ldots\} \\
& \geq 1-\sum_{k=1}^{\infty} \mathrm{P}\{H(k \tau) \geq k n\} \geq 1-\sum_{k=1}^{\infty} \frac{(k \tau)^{k n}}{(k n) !} \\
& \geq 1-\sum_{k=1}^{\infty} \frac{\gamma^{k n}}{\sqrt{2 \pi k n}} \geq 1-\frac{1}{\left(1-\gamma^{-n}\right) \sqrt{2 \pi n}} .
\end{aligned}
$$


Letting $n$ tend to infinity, we obtain

$$
\mathrm{P}\left\{\limsup _{t \rightarrow \infty} \frac{H(t)}{t}<\gamma e\right\}=1 .
$$

Hence, since $\gamma$ can be arbitrary close to 1 ,

$$
\mathrm{P}\left\{\limsup _{t \rightarrow \infty} \frac{H(t)}{t} \leq e\right\}=1
$$

which is the announced result.

Finally the second assertion of theorem 2.1 is a straight consequence of the superadditivity of the function $\mathrm{E} H(t)$,

$$
\mathrm{E} H(s+t) \geq \mathrm{E} H(s)+\mathrm{E} H(t)
$$

which, by a well known lemma, implies the existence of $\lim _{t \rightarrow \infty} \frac{H(t)}{t}$.

Theorem 2.1 is completely proved.

\section{The general case: $\lambda>0, \mu>0$}

In this section leaves can be deleted and the main problem is to find the exact conditions for the process to be ergodic or transient.

With this in view, define the lifetime $\tau(v)$ of an arbitrary vertex $v$, which measures the length of the time interval between the birth and the death of $v$ (for consistency $\tau(v)=\infty$ if $v$ is never erased).

Lemma 3.1. All vertices, but the root, have the same lifetime distribution $p(t)$, which satisfies the following system (S)

$$
\begin{aligned}
& \beta(t)=\mu \exp \left\{-\lambda \int_{0}^{t}(1-p(x)) d x\right\} \\
& \beta(t)=\frac{d p(t)}{d t}+\int_{0}^{t} \beta(t-y) d p(y),
\end{aligned}
$$

with the initial condition $p(0)=0$. 
Proof. Let $v$ be a particular vertex of $G(t)$ and consider the random subtree with root $v$. Its evolution does not depend on anything below $v$, as long as $v$ exists. Therefore all these subtrees are identically distributed and, accordingly, their vertices have the same lifetime distribution.

To capture more precisely the evolution of the process, we introduce two important random variables associated with each vertex $v$ :

- $t_{v}$, the proper time of $v$, such that $v$ appears at $t_{v}=0$;

- $X\left(t_{v}\right)$, the number of direct descendants of $v$ (i.e. who are located at a distance 1 of $v)$.

At rate $\lambda$, a vertex $v$ produces descendants whose lifetimes are independent, with the common distribution $p(t)$. As soon as $X\left(t_{v}\right)=0, v$ can die at rate $\mu$, in which case the process of production stops.

Next, consider a companion process $\widetilde{X}\left(t_{v}\right)$, which is a slight modification of $X\left(t_{v}\right)$ in that, instead of deleting $v$, a $\mu$-event occurs without stopping the production of descendants. Let $\theta$ denote the epoch of the first $\mu$-event. Observing that $p(t)$ is just the probability distribution of the random variable $\theta$, it is not difficult to construct $X\left(t_{v}\right)$ from $\widetilde{X}\left(t_{v}\right)$ on the same probability space. As a matter of fact,

$$
X\left(t_{v}\right)= \begin{cases}\tilde{X}\left(t_{v}\right), & \text { if } t_{v} \leq \theta \\ 0, & \text { if } t_{v} \geq \theta\end{cases}
$$

For any fixed $t_{v}$, we write down a sum of conditional probabilities, expressing the fact that $v$ had exactly $k$ descendants, who all have died in $\left[0, t_{v}\right]$, their birth-times being independent and uniformly spread over $\left.\left[0, t_{v}\right]\right)$. This yields at once equation (3.1), since

$$
\mathrm{P}\left\{\tilde{X}\left(t_{v}\right)=0\right\}=\sum_{k=0}^{\infty} \frac{e^{-\lambda t_{v}}\left(\lambda t_{v}\right)^{k}}{k !}\left(\int_{0}^{t_{v}} \frac{p(x) d x}{t_{v}}\right)^{k}=\exp \left\{-\lambda \int_{0}^{t_{v}}(1-p(x)) d x\right\} .
$$

By means of a regenerative argument, it is also possible to rewrite the above probability in another way, starting from the decomposition

$$
\mathrm{P}\left\{\tilde{X}\left(t_{v}\right)=0\right\}=\mathrm{P}\left\{\tilde{X}\left(t_{v}\right)=0, \theta \geq t_{v}\right\}+\mathrm{P}\left\{\tilde{X}\left(t_{v}\right)=0, \theta<t_{v}\right\} .
$$


In fact, we have the trite relations

$$
\left\{\begin{array}{l}
\mathrm{P}\{\theta \in(y, y+d y)\}=d p(y), \\
\frac{d p\left(t_{v}\right)}{d t}=\mu \mathrm{P}\left\{\tilde{X}\left(t_{v}\right)=0, \theta \geq t_{v}\right\}, \\
\mathrm{P}\left\{\tilde{X}\left(t_{v}\right)=0, \theta<t_{v}\right\}=\mathrm{P}\left\{\tilde{X}\left(t_{v}-\theta\right)=0, \theta<t_{v}\right\}
\end{array}\right.
$$

which yield in particular,

$$
\mathrm{P}\left\{\widetilde{X}\left(t_{v}\right)=0, \theta<t_{v}\right\}=\int_{0}^{t_{v}} \mathrm{P}\left\{\widetilde{X}\left(t_{v}-y\right)=0\right\} d p(y) .
$$

Hence, putting $\beta(t) \stackrel{\text { def }}{=} \mu \mathrm{P}\left\{\widetilde{X}\left(t_{v}\right)=0\right\}$, one sees that (3.3) corresponds term by term to (3.2). The proof of the lemma is concluded.

\section{Theorem 3.1.}

(A) The Markov chain $G$ is ergodic if, and only if,

$$
\rho \stackrel{\text { def }}{=} \frac{\lambda}{\mu} \leq \frac{1}{e}
$$

(B) When the sytem is ergodic, the mean lifetime $m \stackrel{\text { def }}{=} \mathrm{E}\left(\tau_{v}\right)$ is given by

$$
m=\frac{r}{\lambda},
$$

where $r$ denotes the smallest root of the equation

$$
r e^{-r}=\rho .
$$

(C) When $\rho>\frac{1}{e}$, then the system is transient. More precisely, $\lim _{t \rightarrow \infty} p(t) \stackrel{\text { def }}{=} \ell<1$, where $\ell$ might be extracted from the solution of the integral equation (3.14).

The proof of the theorem is spread over the next three sections. 


\subsection{Ergodicity}

Relying on the standard theory of Markov chains with countable state space (see [3], vol. I), we claim the system ergodic if, and only if, $m<\infty$. As a matter of fact, the $\mu$-events are regeneration points for the process $\widetilde{X}(t)$, which represents exactly the number of descendants of the root $V_{0}$. Hence when $\mathrm{E}(\tau(v))<\infty$ (i.e. $\beta(\infty)>0$ ), the event $\{\widetilde{X}(t)=0\}$ has a positive probability, since $\theta$ has the same distribution as $\tau(v)$, so that $G$ is ergodic. Conversely, if $\mathrm{E}(\tau(v)))=\infty$ then $\widetilde{X}(t)$ is transient and so is $G$.

To show the necessity of condition (3.4), suppose $G$ is ergodic. Then, by (3.1), there exists the limit

$$
\lim _{t \rightarrow \infty} \beta(t) .
$$

For an arbitrary positive function $f$, denote by $f^{*}$ its ordinary Laplace transform

$$
f^{*}(s) \stackrel{\text { def }}{=} \int_{0}^{\infty} e^{-s t} f(t) d t, \quad \Re(s) \geq 0 .
$$

By (3.1), $e^{-m} \leq \alpha(t) \leq 1$, so that we can apply a limiting relation of Abelian type for Laplace transforms (see e.g. [4]). Hence equations (3.1) and (3.2) - the latter being of Volterra class - yield respectively

$$
\left\{\begin{array}{l}
\lim _{t \rightarrow \infty} \beta(t)=\lim _{s \rightarrow 0} s \beta^{*}(s)=\lim _{s \rightarrow 0} \frac{s^{2} p^{*}(s)}{1-s p^{*}(s)}=\frac{1}{m}, \\
\lim _{t \rightarrow \infty} \beta(t)=\mu e^{-\lambda m}
\end{array}\right.
$$

whence the equality

$$
\rho=\lambda m e^{-\lambda m}
$$

As the function $x e^{-x}$ reaches its maximum $e^{-1}$ at $x=1$, we conclude that $\rho \leq e^{-1}$.

In order to prove the sufficiency of (3.4), we have to get a deeper insight into system (S). There will be done along two main steps.

(a) Although (S) reduces to a second order non-linear integro-differential equation, this does not help much. What is more useful is that all derivatives $p^{(n)}(0), \beta^{(n)}(0)$, 
taken at the the origin in the complex $t$-plane, can be recursively computed for all $n$. This can be checked at once, rewriting (3.1) in the differential form

$$
\frac{d \beta(t)}{d t}+\lambda(1-p(t)) \beta(t)=0 .
$$

Noticing the derivatives $p^{(n)}(0)$ [resp. $\beta^{(n)}(0)$ ] have alternate signs when $n$ varies, it is direct to verify that $\beta$ and $p$ are analytic functions around the origin, and that their respectve power series have a non-zero radius of convergence. The first singularities of $p$ and $\beta$ are on the negative real axis, but not easy to locate precisely. Hence (S) has a solution, which is unique, remarking also that uniqueness is a mere consequence of the Lipschitz character of $\frac{d p(t)}{d t}$ with respect to $\beta$ in the Volterra integral equation (3.2) (see e.g. [1]). En passant, it is worth noting that the solution in the whole complex plane -which is not really needed for our purpose- could be obtained by analytic continuation directly on system (S).

(b) When (3.4) holds, the next stage consists in exhibiting a non-defective probabilistic solution $p(t)$ [necessarily unique by step (a)], with a finite mean $m<\infty$. This is more intricate and will be achieved by constructing a converging iterative scheme.

Consider the system

$$
\left\{\begin{array}{l}
\beta_{0}(t)=\mu, \quad t \geq 0 \\
\beta_{k}(t)=\frac{d f_{k}(t)}{d t}+\int_{0}^{t} \beta_{k}(t-y) d f_{k}(y) \\
\beta_{k+1}(t)=\mu \exp \left\{-\lambda \int_{0}^{t}\left(1-f_{k}(y)\right) d y\right\} \\
f_{k}(0)=0, \forall k \geq 0 .
\end{array}\right.
$$

The second equation in (3.8) is equivalent to

$$
s f_{k}^{*}(s)=\frac{\beta_{k}^{*}(s)}{1+\beta_{k}^{*}(s)},
$$

allowing to derive $f_{k}$ from $\beta_{k}$, by means of any classical inversion formula for Laplace transforms. Hence, the computational algorithm becomes simple:

Step $1: f_{0}(t)=1-e^{-\mu t}$. 
Step 2 : Compute $\beta_{1}(t)=\mu \exp \left[-\rho\left(1-e^{-\mu t}\right)\right]$.

Step 3 : Compute $f_{1}(t)$, then $\beta_{2}(t), f_{2}(t)$, etc.

At each step, the successive $f_{k}$ 's are non-defective probability distributions, with finite means denoted by $c_{k}$. Indeed, one has to check first that the right-hand side of (3.9) is the Laplace transform of a positive measure, since a priori it does not correspond to a completely monotone function, according to the classical definition of [3]. The following easy lemma does answer this question and might be of intrinsic interest.

Lemma 3.2. Let $Q$ be a measure concentrated on $[0, \infty[$ and define

$$
\psi(s) \stackrel{\text { def }}{=} \int_{0}^{\infty} \mu e^{-(\lambda Q(t)+s t)} d t, \quad \Re(s) \geq 0 .
$$

Then $\omega(s) \stackrel{\text { def }}{=} \frac{\psi(s)}{1+\psi(s)}$ is the Laplace transform of a positive measure on $[0, \infty[$, which in addition is a decreasing functional of $Q$.

Proof. Take $\Re(s) \geq \mu$. Then the function $\widetilde{\psi}(s) \stackrel{\text { def }}{=} \psi(s+\mu)$ can be viewed as the the Laplace transform of a positive random variable $U$ having the probability density $\mu e^{-(\lambda Q(t)+\mu t)}$. Thus

$$
\mathrm{P}\{U \leq t\}=\int_{0}^{t} \mu e^{-(\lambda Q(t)+\mu t)} d t \leq 1-e^{-\mu t} \leq 1,
$$

and the following expansion holds

$$
\omega(s)=\sum_{k=0}^{\infty}(-1)^{k} \widetilde{\psi}^{(k+1)}(s-\mu),
$$

where the modulus of the $k$-th term of the series stands for the transform of the $(k+1)$-fold convolution of $U$. A function being uniquely determined [up to values in a set of measure zero] by the values of its Laplace transform in the region $\Re(s) \geq \mu$, the conclusion of the lemma follows immediately by using elementary properties of alternate series, together with the contraction (3.10).

The scheme (3.8) enjoys two nice properties.

(i) It is monotone decreasing. Suppose $f_{k}(t) \leq f_{k-1}(t)$, which is in particular true for $k=1$. Then the third equation of (3.8) implies $\beta_{k+1}(t) \leq \beta_{k}(t)$, so that by 
lemma $3.2 f_{k+1}(t) \leq f_{k}(t)$. The probabilistic interpretation is clear, remarking that, in the third equation of (3.8), $\beta_{k}(t) / \mu$ is simply the probability of being empty for an $\mathrm{M} / \mathrm{G} / \infty$ queue with service time distribution $f_{k}$.

So, the positive sequences $\left\{f_{k}(t), k \geq 0\right\}$ and $\left\{\beta_{k}(t), k \geq 0\right\}$ are uniformly bounded and non-increasing for each fixed $t$. Consequently,

$$
p(t)=\lim _{k \rightarrow \infty} \downarrow f_{k}(t) \quad \text { and } \quad \beta(t)=\lim _{k \rightarrow \infty} \beta_{k}(t)
$$

form the unique solutions of $(\mathrm{S})$.

(ii) Letting $r_{k} \stackrel{\text { def }}{=} \lambda c_{k}$ and combining the two main equations of (3.8), we get

$$
r_{k+1}=\rho e^{r_{k}}, \forall k \geq 0, \quad \text { with } r_{0}=\rho .
$$

For $\rho \leq e^{-1}$, the $r_{k}$ 's form an increasing sequence of positive real numbers, with a finite positive limit $r$ satisfiying equation (3.5). Since $1-f_{k}(t)$ is an increasing sequence of positive functions, the theorem of Beppo Levi ensures the equality

$$
\int_{0}^{\infty}(1-p(t)) d t=\lim _{k \rightarrow \infty} \int_{0}^{\infty}\left(1-f_{k}(t)\right) d t=\lim _{k \rightarrow \infty} c_{k}=\frac{r}{\lambda} .
$$

We have thus proved points (A) and (B) of the theorem

Remarks In the scheme (3.8), the initial condition $\beta_{0}(t)=\mu$ is tantamount to say that here was implicitly a ficticious function, say $f_{-1}$ satisfying $f_{-1}(t)=1, \forall t \geq 0$.

But we could have also considered the scheme

$$
\left\{\begin{array}{l}
\gamma_{0}(t)=\mu e^{-\lambda t}, \quad t \geq 0 \\
\gamma_{k}(t)=\frac{d g_{k}(t)}{d t}+\int_{0}^{t} \gamma_{k}(t-y) d g_{k}(y), \\
\gamma_{k+1}(t)=\mu \exp \left\{-\lambda \int_{0}^{t}\left(1-g_{k}(y)\right) d y\right\} \\
g_{k}(0) \quad=0, \forall k \geq 0
\end{array}\right.
$$

which differs from (3.8) only by its first equation, but this difference is crucial and corresponds to a ficticious function $g_{-1}(t)=0, \forall t \geq 0$. 
The scheme (3.12) enjoys the following properties: it is increasing; the distribution $g_{k}$ are defective, and they dominate defective exponential distributions with Laplace transforms of the form $\frac{u_{k}}{v_{k}+s}$; under condition (3.4),

$$
\lim _{k \rightarrow \infty} u_{k}=\lim _{k \rightarrow \infty} v_{k}=\frac{\lambda}{r}
$$

and $g_{k}$ converges in $L_{1}$ to the proper distribution $p$. We shall return to (3.8) in the next section.

\subsection{Transience}

After needlessly complicated attempts, it turns out that the classification of the process when $\rho>e^{-1}$ can be obtained rather straightforwardly from analytic arguments.

Recalling that $\ell=\lim _{t \rightarrow \infty} p(t)$, we define

$$
\left\{\begin{array}{l}
\varepsilon(t) \stackrel{\text { def }}{=} \lambda \int_{0}^{t}(\ell-p(x)) d x, \quad \text { with } \frac{d \varepsilon(t)}{d t \mid t=0}=\lambda \ell, \\
\varphi(s) \stackrel{\text { def }}{=} \int_{0}^{\infty} \exp [-(\varepsilon(t)+(\lambda(1-l)+s) t)] d t
\end{array}\right.
$$

Then the following functional equation holds

$$
\frac{s^{2} \varepsilon^{*}(s)}{\lambda}=\frac{\ell+\mu(\ell-1) \varphi(s)}{1+\mu \varphi(s)} .
$$

Assume now $l=1$. Then (3.14) yields

$$
s^{2} \varepsilon^{*}(s)\left[1+\mu \int_{0}^{\infty} \exp [-(\varepsilon(t)+s) t] d t\right]=\lambda
$$

Letting now $s$ tend to 0 in (3.15) and applying classical properties of Laplace transforms [already used in (3.6)], we get the equality

$$
\lim _{t \rightarrow \infty}[\varepsilon(t) \exp (-\varepsilon(t))]=\rho
$$

which is possible only if $\rho \leq e^{-1}$. Thus when $\rho>e^{-1}$ one has necessarily $\ell<1$, and the system is transient. 
The last point is the exact computation of $\ell$, which turns out to be an intricate problem. Without presenting a complete solution in this report [although one can hardly expect more than an approximate formula], we shall nonetheless present the main lines of two possible ways of tackling this question.

The first one might be to proceed by analytic continuation on the functional equation (3.14). Indeed, from the definition (3.13) the right-hand side member of (3.14) can be analytically continued to the region $\Re(s)<-\lambda(1-\ell)$, thus rendering possible an analysis of singularities, from where the values $\ell$ and $L \stackrel{\text { def }}{=} \lim _{t \rightarrow \infty} \varepsilon(t)$ could be obtained.

The second method is more constructive and relies on the iterative scheme (3.12), which is convergent for all $\rho$. In this scheme, the functions $g_{k}(t), k \geq 0$, are defective, their limit being proper if and only if $\rho \leq e^{-1}$. When $\rho>e^{-1}$, the limiting function $p(t)$ is still defective and

$$
\lim _{t \rightarrow \infty} p(t)=\lim _{k \rightarrow \infty} \lim _{t \rightarrow \infty} g_{k}(t)=\ell<1 .
$$

Hereafter we derive a lower bound on $l$, and an upper bound on $L$ in showing by induction that, for each $k, g_{k}(t)$ dominates an exponential distribution.

Take the variable $s$ real positive and assume

$$
g_{0}(t) \geq \frac{a_{k}}{b_{k}}\left[1-e^{-b_{k} t}\right]
$$

which is in particular true for $k=0$, since $g_{0}(t)=\frac{\mu}{\lambda+\mu}\left[1-e^{-(\lambda+\mu) t}\right]$. Then the third equation of (3.12) yields, after some calculus,

$$
\begin{aligned}
\gamma_{k+1}(t) & \geq \mu \exp \left[-\lambda\left(1-\frac{a_{k}}{b_{k}}\right) t+\frac{\lambda a_{k}}{b_{k}^{2}}\left(e^{-b_{k} t}-1\right)\right] \\
& \geq \mu \exp \left(\frac{-\lambda a_{k}}{b_{k}^{2}}\right) \exp \left[-\lambda\left(1-\frac{a_{k}}{b_{k}}\right) t\right]
\end{aligned}
$$

so that

$$
s g_{k+1}^{*}(s)=\frac{\gamma_{k+1}^{*}(s)}{1+\gamma_{k+1}^{*}(s)} \geq \frac{\mu \exp \left(\frac{-\lambda a_{k}}{b_{k}^{2}}\right)}{\mu \exp \left(\frac{-\lambda a_{k}}{b_{k}^{2}}\right)+\lambda\left(1-\frac{a_{k}}{b_{k}}\right)+s} .
$$

Hence the induction hypothesis holds, provided the sequences $a_{k}, b_{k}, k \geq 0$, satisfy the recursive relationships 


$$
\left\{\begin{array}{l}
a_{k+1}=\mu \exp \left(\frac{-\lambda a_{k}}{b_{k}^{2}}\right) \\
b_{k+1}=a_{k+1}+\lambda\left(1-\frac{a_{k}}{b_{k}}\right) .
\end{array}\right.
$$

Setting $a \stackrel{\text { def }}{=} \lim _{k \rightarrow \infty} a_{k}$ and $b \stackrel{\text { def }}{=} \lim _{k \rightarrow \infty} b_{k}$ in $(3.17)$, it is not difficult to see that

$$
\frac{a}{b}= \begin{cases}1, & \text { if } \quad \rho \leq e^{-1}, \\ x, & \text { if } \quad \rho>e^{-1}\end{cases}
$$

where $x \leq 1$ is the root of the equation

$$
x e^{x}=\frac{1}{\rho} .
$$

Thus in the transient case the following bounds hold

$$
\left\{\begin{array}{l}
\ell \leq x \\
\int_{0}^{\infty} t d p(t)=\int_{0}^{\infty}(\ell-p(t)) d t=\frac{L}{\lambda} \leq \frac{a}{b^{2}}=\frac{x}{\lambda}
\end{array}\right.
$$

and it is interesting to compare (3.19) with the results in theorem 3.1.

The proof of theorem 3.1 is terminated.

Subsidiary comments The method of schemes to analyze non-linear operators in a probabilistic contex is extremely powerful (see e.g. [2] for problems related to systems in thermodynamical limit), and in some sense deeply related to the construction of Lyapounov functions. Up to sharp technicalities, the schemes (3.8) and (3.12) can be exploited to derive precise information about the speed of convergence when $t \rightarrow \infty$, for any $\rho, 0<\rho<\infty$, and when pushing exact computations slightly farther, one perceives underlying relationships with some complicated continued fractions. Finally, we note that the question of transience could be studied from a large deviation point of view, by considering $\varepsilon(t)$ as the member of a family indexed by the parameter $\left(\rho-e^{-1}\right)$. 
Acknowledgements The authors thank V.A. Malyshev for bringing this problem to their attention, Th. Deneux and J.-M. Lasgouttes for useful numerical experiments and fruitful discussions.

\section{References}

[1] H. Cartan (1977) Cours de calcul différentiel, Hermann, Collection Méthodes.

[2] F. Delcoigne And G. Fayolle (1999) Thermodynamical limit and propagation of chaos in polling systems, Markov Processes and Related Fields, 5 (1), pp. 89-124.

[3] W. Feller (1971) An Introduction to Probability Theory and its Applications, Vol. I and II, Wiley.

[4] B.A Fuchs And V.I. Levin (1961) Functions of a Complex Variable, Vol. II, Pergamon Press.

[5] M. KRIKun (2000) Height of a random tree, Markov Processes and Related Fields, 6 (2), pp. 135-146.

[6] H.M. Mahmoud (1992) Evolution of Random Search Trees, Wiley-Intersciences Series.

$\mathrm{RR} \mathrm{n}^{\circ} 4331$ 


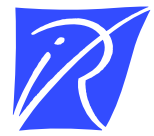

Unité de recherche INRIA Rocquencourt

Domaine de Voluceau - Rocquencourt - BP 105 - 78153 Le Chesnay Cedex (France)

Unité de recherche INRIA Lorraine : LORIA, Technopôle de Nancy-Brabois - Campus scientifique 615, rue du Jardin Botanique - BP 101 - 54602 Villers-lès-Nancy Cedex (France)

Unité de recherche INRIA Rennes : IRISA, Campus universitaire de Beaulieu - 35042 Rennes Cedex (France)

Unité de recherche INRIA Rhône-Alpes : 655, avenue de l'Europe - 38330 Montbonnot-St-Martin (France)

Unité de recherche INRIA Sophia Antipolis : 2004, route des Lucioles - BP 93 - 06902 Sophia Antipolis Cedex (France)

INRIA - Domaine de Voluceau - Rocquencourt, BP 105 - 78153 Le Chesnay Cedex (France)

http://www.inria.fr

ISSN 0249-6399 